\title{
A Preliminary Clinical Evaluation of a Topical Product for Reducing Slight Rosacea Imperfections
}

This article was published in the following Dove Press journal:

Clinical, Cosmetic and Investigational Dermatology

\author{
Daniele Maggioni' \\ Annamaria Cimicata' \\ Antonella Praticò' \\ Roberta Villa' \\ Ferdinando Marco Bianchi $\mathbb{D D}^{1,2}$ \\ Silvia Busoli Badiale' \\ Claudio Angelinetta' \\ 'Bio Basic Europe Srl, Milan, Italy; \\ ${ }^{2}$ Dermo-Cosmetic and Medical R\&D \\ Center of Bio Basic Europe Srl, Milan, \\ Italy
}

\begin{abstract}
Introduction: Rosacea is a chronic multifactorial skin disorder mainly affecting facial skin with an estimated prevalence of about $5 \%$ worldwide. Its main symptoms, occurring early during pathology development, are skin dehydration, redness, erythema, and telangiectasia. Given the lack of a resolutive cure, therapeutic approaches able to relieve the main symptoms are needed.
\end{abstract}

Purpose: The aim of this research article is to evaluate the beneficial effect of a topical product (Serum BK46) on rosacea symptoms.

Patients and Methods: A monocentric single-arm, non-blinded study was performed to assess the clinical effect of Serum BK46 in relieving the main symptoms of rosacea: skin dryness, increased trans epidermal water loss (TEWL), redness, and abnormal vascularization. Twenty patients with mild to moderate rosacea were enrolled in the study and asked to apply the product twice per day for 56 days. Skin moisturization, TEWL, and erythema index were instrumentally assessed at baseline and following $24 \mathrm{~h}$ and 14, 28 and 56 days of treatment. Clinical parameters, including redness and telangiectasia imperfection visibility, were evaluated on a 5-point scale by a specialized dermatologist at baseline and after 14, 28, and 56 days of treatment. Finally, the visibility of vessel diameter was evaluated at baseline and after 28 and 56 days of treatment.

Results: Serum BK46 application restored skin hydration and prevented the loss of water by the skin. Long-term treatment with Serum BK46 significantly reduced skin redness, erythema index, and the visibility of telangiectasia imperfections and superficial vessels. The investigated product's clinical effect was demonstrated by both instrumental and clinical evaluation. Furthermore, Serum BK46 was completely tolerated and no adverse effects were recorded.

Conclusion: The moisturizing and skin barrier restoring action of Serum BK46 has been clearly proven in patients displaying mild to moderate rosacea; thus, this product is a good candidate for rosacea treatment.

Keywords: rosacea, topical product, transepidermal water loss, skin redness, telangiectasia

\section{Introduction}

Rosacea is a common inflammatory skin disorder, affecting prevalently the nose, chin, and central forehead and in some cases the periocular areas. ${ }^{1}$ Its etiology and pathophysiology are still largely unknown.

Rosacea is a diffuse pathology affecting more than 15 million patients in USAthe only, while its prevalence is estimated between $5 \%$ and $10 \%$ worldwide. ${ }^{1,2}$

Furthermore, the incidence of rosacea is increasing in developed countries, probably because of the aging populations. Rosacea is more prevalent in fairskinned people, especially among European Caucasians, but it has also been
Correspondence: Annamaria Cimicata

Tel +39024155729

Fax +390242174243

Email annamariacimicata@libero.it 
reported in people of other ethnicities. ${ }^{3}$ Females and males are equally affected, although the manifestations of the pathology may vary according to gender. ${ }^{4}$ The highest prevalence of the pathology is found among people in the age range between 45 and 60 years. ${ }^{1}$

Although rosacea etiology remains not completely understood, it seems to be multifactorial, comprising both a genetic predisposition and environmental factors that can promote an exaggerated vasodilation and water loss. ${ }^{5}$ For instance, rosacea may be initiated or aggravated by several trigger factors, such as cold, heat, ultraviolet irradiation, and dermal or oral exposure to chemicals. ${ }^{4}$

Based on morphological characteristics, rosacea may be classified into four groups: ocular, erythethematotelangiectatic, papulopustular, and phymatous. Ocular rosacea affects the periocular zone, whereas erythematotelangiectatic, phytamous and papulopustular rosacea arise in the middle of the face and they primarily include telangiectasia, erythema, and flushing. ${ }^{6}$ Primary symptoms are usually associated with secondary features such as burning, itching, and stinging sensation on the face. ${ }^{7}$

The unknown etiology and the intrinsic heterogeneity of manifestations of rosacea have hampered the efficacy of treatments. In fact, although several therapeutic options such as topical application of vasoconstrictive or antiinflammatory creams or laser treatments are used, a proper cure for this disorder does not exist and current treatments usually address and manage only some symptoms of the pathology. ${ }^{6}$

Although it is not considered a severe pathology, the psycho-social impact of rosacea is often underrated; in fact, the pathology deeply impacts patients' quality of life, as the negative self-perception of the patient may lead to psychological co-morbidity. For instance, epidemiological data reported an increased depression rate among patients with rosacea. ${ }^{3}$

Pharmaceutical research for novel rosacea treatment is somewhat limited if compared with research in other inflammatory skin diseases. It is unlikely that a single therapeutic modality will result in complete and longlasting resolution of rosacea. In mild to moderate disease, topical approaches are the first-line treatment and they comprise a broad spectrum of formulations including metronidazole, azelaic acid, clindamycin lotion, permethrin 5\% cream, tretinoin cream, 10\% sulfacetamide with sulfur $(5 \%)$, and benzoyl peroxide alone or in combination with erythromycin or clindamycin. ${ }^{8}$ These approaches are not always completely effective; moreover, these pharmacological treatments can be used for a limited time only.

The Serum BK46 applied in this study is a topical formulation based on skin moisturizing, relieving, and protecting agents, that help to increase the cutaneous barrier function, thus preventing skin dehydration and protecting the skin from all the environmental factors that may promote and exacerbate the pathology symptoms. In particular, Serum BK46 contains a combination of ingredients such as potassium azeloyl diglycinate, squalane, dipotassium glycyrrhizate, Aloe barbadensis leaf juice, sodium hyaluronate, polyacrylate crosspolymer-6, and xanthan gum, that synergistically act to normalize and relieve rosacea symptoms.

Potassium azeloyl diglycinate, thanks to the presence of glycine, possesses a moisturizing effect and helps to maintain skin hydration levels, so the affected skin stays moisturized, allowing the physiological healing process through restoring skin balance conditions on the compromised area. Squalane is an oily substance that acts as a lubricant on the skin surface; it functions as an emollient for topical application in creams, lotions, ointments, lipsticks, and other cosmetics. Dipotassium glycyrrhizate is a skin conditioning/humectant (as per cosmetic indications) and it is used in cosmetic formulations (FDA 2002) at a maximum concentration of 1\% (CTFA 2003). Xanthan gum is a polysaccharide used as an emulsion stabilizer and gelling agent and it is globally recognized as a film former. Hyaluronic acid is a well-known humectant and skin-conditioning polysaccharide distributed widely in the extracellular matrix of human connective tissue. Due to high molecular weight, it is considered to help the creation of a moisturizing film on the skin surface. Polyacrylate crosspolymer-6 is a high molecular weight polymer able to deposit over the outer layers of the skin/ mucosae. It works with other ingredients and helps to create a barrier to restoring skin and preventing water loss and hydrating tissues.

Aloe barbadensis leaf juice is the juice expressed from the leaves of the aloe, Aloe barbadensis, Liliaceae. It functions as a film former, humectant, skin-conditioning agent (emollient).

Given the high incidence and the psycho-social impact of the pathology, rosacea treatment represents an unmet clinical need; therefore, the aim of this pilot study was to evaluate the effect of Serum BK46 in the alleviation of rosacea symptoms. In particular, the effect of Serum BK46 in reducing rosacea signs has been analyzed by measurement of skin 
moisturization, transepidermal water loss (TEWL), capillary diameter and erythema index and by clinical visual evaluation of clinical parameters such as redness and visibility of telangiectasia associated skin imperfections.

\section{Materials and Methods}

A single-site study of Serum BK46 for the treatment of rosacea was conducted in 2017 at Dermo-Cosmetic and Medical R\&D Center of Bio Basic Europe (Milan, Italy). All subjects, enrolled in a single cohort, provided written informed consent prior to entering into the study. The trial was run in accordance with the Declaration of Helsinki (2013) and with principles of good clinical practices. The study protocol was approved by the internal ethics committee of the Medical R\&D Center of Bio Basic Europe. According to the structure of the study, both patients and examiners were not blinded. Data were obtained, recorded, and processed in accordance with the International Conference on Harmonization of Technical Requirements for Registration of Pharmaceuticals for Human Use (ICH) guidelines for good clinical practice.

Healthy subjects $(n=20)$, with an age between 30 and 65 years, have been selected for the investigation. Following a baseline clinical visit, patients with dry skin, reddened skin, and imperfections caused by telangiectasia (mild to moderate rosacea) were included in the study. Patients were excluded from experimentation in the case of dermatopathies, if they consume oral or topic drugs, or if they complained of psychological diseases. Moreover, patients were required to not change their usual daily routine. Before taking any measurement, the patients were accommodated in a room with a controlled atmosphere $\left(20^{\circ} \mathrm{C}\right.$, RH $\left.40-60 \%\right)$ for a period of time.

We hereby acknowledge the company Biokosmes s.r.l. for providing the product involved in this study.

\section{Method of Application of the Test Samples}

Samples of the tested product have been applied on the face skin twice per day (in the morning and in the evening) after routine cleansing, for 56 consecutive days.

At the beginning and throughout the entire experimentation, at selected time points (after 24 h, 14 days, 28 days and 56 days of treatment), instrumental evaluations of skin moisturization, transepidermal water loss (TEWL), and erythema index were conducted. In addition, clinical evaluation of skin redness and telangiectasia imperfections were performed at the beginning of the study and after 14,28 , and 56 days of treatment.

\section{Instrumental Evaluation of Skin Parameters}

Skin moisturization was measured by the use of a corneometer ${ }^{\circledR} \mathrm{CM} 825$ (Courage and Khazaka, Koln, Germany). Briefly, the corneometer measures the electric capacity of the skin surface that is related to skin moisture: both electric capacity and the conductance of biological tissue change according to the water content, ie, they increase if the water content increases. This instrument translates the electrical parameters in moisturization units (scale: 0-130).

The TEWL was evaluated through the TEWAMETER ${ }^{\circledR}$ TM 300 (Courage and Khazaka, Koln, Germany); this instrument indicates the TEWL, in terms of quantity of evaporated water for the considered skin unit. The method measures the gradient of steam tension between two electrodes located at different distances from the skin surface. The method allows the corneal layer integrity and functionality of the skin barrier to be evaluated.

The erythema index was analyzed by using the Mexameter MX18 (Courage and Khazaka, Koln, Germany), which measures the absorption capacity of the skin. The instrument emits light at two different wavelengths and measures the light reflected by the skin. Since the amount of light emitted is known, the quantity of light absorbed by the skin can be calculated. Two wavelengths were used: 568 and $660 \mathrm{~nm}$, corresponding to the absorption peak of hemoglobin and color influencers such as melanin bilirubin, respectively. The erythema index was calculated from the digitized results obtained in a 0-999 scale as a function of skin absorption at $568 \mathrm{~nm}$ minus absorption at $660 \mathrm{~nm}$.

Blood capillary diameter measurements were performed by using VIDEOCAP $^{\circledR}$, Video-Capillaroscopy with a tube (D-S Medica, Milan, Italy). The instrument measures the diameter of vessels at the level of arboreal telangiectasias and capillary loops' linear branches, clearly visible during the videodermatoscopic evaluation.

The instrument is put on the specific skin areas and images are recorded and subsequently analyzed at $200 \times$ magnification. Vessel diameter is calculated using VIDEOCAP ${ }^{\circledR}$ software and expressed in $\mathrm{mm}$. Each recorded measure is the average of 10 measurements performed on 10 vessels.

In addition, images of the telangiectasia lesions were taken by the use of Miravex Antera 3D.

\section{Clinical Evaluation}

Clinical evaluation was performed through visual assessment by a dermatologist and the results were recorded 
according to the rosacea scoring system as reported by Wilkin and coworkers ${ }^{1}$ with minor modifications, so that primary signs of rosacea were graded as absent, slight, mild, severe, and very severe in a range from 1, very severe, to 5, absent. Parameters used to evaluate skin redness and imperfection visibility and their scoring system are reported in Table 1. In Table 2 are shown the comparison of clinical evaluation of rosacea symptoms (skin redness and telangiectasia imperfection visibility) score at baseline (before treatment $=\mathrm{T} 0$ ) and after 14, 28, and 56 days of Serum BK46 application.

\section{Patient-Reported Outcome}

At the end of treatment (T56) patients were asked to fulfill an end of study questionnaire, which evaluated the patient's opinions about the product's effects, with particular focus on the feeling of skin dryness, skin burning, and redness. This self-evaluation was performed according to a VNS (visual numeric scale) scale where 0 is the minimum value and 10 is the maximum value. Scores above or equal to 7 have been considered as positive.

\section{Statistical Evaluation}

Statistical analysis of short term (24 h) Serum BK46 effect on skin moisturization was performed by non-parametric Wilcoxon signed-rank test.

Table I Clinical Evaluation Parameters

\begin{tabular}{|c|c|c|}
\hline Redness & Score & Description \\
\hline Very evident & I & Very evident skin redness \\
\hline Evident & 2 & Evident skin redness \\
\hline Moderate & 3 & Moderately visible skin redness \\
\hline Slight & 4 & Slightly visible skin redness \\
\hline Absent & 5 & No skin redness \\
\hline $\begin{array}{l}\text { Telangiectasia } \\
\text { imperfection } \\
\text { visibility }\end{array}$ & Score & Description \\
\hline Very evident & I & $\begin{array}{l}\text { Very evident imperfections } \\
\text { caused by telangiectasia }\end{array}$ \\
\hline Evident & 2 & $\begin{array}{l}\text { Evident imperfections caused by } \\
\text { telangiectasia }\end{array}$ \\
\hline Moderate & 3 & $\begin{array}{l}\text { Moderately visible imperfections } \\
\text { caused by telangiectasia }\end{array}$ \\
\hline Slight & 4 & $\begin{array}{l}\text { Slightly visible imperfection } \\
\text { caused by telangiectasia }\end{array}$ \\
\hline Absent & 5 & $\begin{array}{l}\text { No visible imperfection caused } \\
\text { by telangiectasia }\end{array}$ \\
\hline
\end{tabular}

Statistical evaluation of long-term effect for instrumental parameters (skin moisturization, TEWL, erythema index) was performed using analysis of variance by oneway repeated-measures ANOVA, after assumption verification. When the test results were statistically significant, the Student's $t$-test Bonferroni correction was applied as a post hoc test to compare the differences among different timepoint groups. The long-term effect was assessed separately from the short-term effect and T24 has been measured separately from T14, T28, and T56 where the ANOVA was performed.

Statistical evaluation of the long-term effect on vessel diameters and on clinical data (telangiectasia imperfection visibility and skin redness) obtained at different time points was performed by the non-parametric Friedman test followed by Wilcoxon signed-rank Bonferroni corrected test.

The calculation of the sample size for this pilot study was not performed; however, the sample size was defined based on a previously published study on the efficacy of rosacea treatment. ${ }^{9-11}$

\section{Results}

The study included 20 subjects with rosacea on both sides of the face and all patients enrolled successfully completed the study. Each subject received Serum BK46 twice per day, with the application on skin face for 56 days.

The Serum BK46 effect was evaluated instrumentally by measuring the hydration of the skin by the use of a corneometer.

The increase of skin moisturization promoted by Serum BK46 was already evident following 24-h application, in fact, as illustrated in the graph of Figure 1, a significant increase in skin hydration was observed at $\mathrm{T} 24 \mathrm{~h}$ if compared with $\mathrm{T} 0$, as the medians of moisturization values were 42.5 and 45.5 , respectively ( $p<0.01$ Wilcoxon signed-rank test vs T0). The other parameters evaluated, erythema index and skin redness, did not show any significant variation after short treatment $(24 \mathrm{~h})$, data not shown.

In long-term treatment, the hydration index steadily increased from T14 until the end of the treatment. There was a significant difference between skin hydration at baseline and after product application; in fact, the mean skin moisturization index was $42.5 \pm 3.9$ at T0 and climbed up to $44.1 \pm 3.7 ; 47.6 \pm 3.5$ and $49.7 \pm 3.5$ following 14,28 , and 56 days of treatment ( $p$-value $<0.01)$. The skin moisturization progress during the study is shown in the graph of Figure 2. 
Table 2 Clinical Evaluation of Rosacea Symptoms (Skin Redness and Telangiectasia Imperfection Visibility), Score at Baseline (Before Treatment $=$ T0) and After 14, 28, and 56 Days of Serum BK46 Application

\begin{tabular}{|c|c|c|c|c|}
\hline \multirow[t]{3}{*}{ Grade } & \multicolumn{4}{|c|}{ Skin Redness } \\
\hline & TO & TI4 & T28 & T56 \\
\hline & $\%(n)$ & $\%(n)$ & $\%(n)$ & $\%(n)$ \\
\hline $\begin{array}{l}\text { Very evident } \\
\text { (very evident skin redness) }\end{array}$ & $25 \%(5)$ & $20 \%(4)$ & $10 \%(2)$ & $10 \%(2)$ \\
\hline $\begin{array}{l}\text { Evident } \\
\text { (evident skin redness) }\end{array}$ & $60 \%(12)$ & $50 \%(10)$ & $50 \%(10)$ & $40 \%(8)$ \\
\hline $\begin{array}{l}\text { Moderate } \\
\text { (moderately visible skin redness) }\end{array}$ & $15 \%(3)$ & $30 \%(6)$ & $30 \%(6)$ & $30 \%(6)$ \\
\hline $\begin{array}{l}\text { Slight } \\
\text { (slightly visible skin redness) }\end{array}$ & $0 \%(0)$ & $0 \%(0)$ & $10 \%(2)$ & $20 \%(4)$ \\
\hline $\begin{array}{l}\text { Absent } \\
\text { (no skin redness) }\end{array}$ & $0 \%(0)$ & $0 \%(0)$ & $0 \%(0)$ & $0 \%(0)$ \\
\hline \multirow[t]{3}{*}{ Grade } & \multicolumn{4}{|c|}{ Telangiectasia Imperfection Visibility } \\
\hline & TO & TI4 & T28 & T56 \\
\hline & $\%(n)$ & $\%(n)$ & $\%(n)$ & $\%(n)$ \\
\hline $\begin{array}{l}\text { Very evident } \\
\text { (very evident imperfections caused by telangiectasia) }\end{array}$ & $20 \%(4)$ & $20 \%(4)$ & $15 \%(3)$ & $5 \%(1)$ \\
\hline $\begin{array}{l}\text { Evident } \\
\text { (evident imperfections caused by telangiectasia) }\end{array}$ & $55 \%(11)$ & $55 \%(I I)$ & $45 \%(9)$ & $50 \%(10)$ \\
\hline $\begin{array}{l}\text { Moderate } \\
\text { (moderately visible imperfections caused by telangiectasia) }\end{array}$ & $20 \%(4)$ & $20 \%(4)$ & $25 \%(5)$ & $25 \%(5)$ \\
\hline $\begin{array}{l}\text { Slight } \\
\text { (slightly visible imperfections caused by telangiectasia) }\end{array}$ & $5 \%(1)$ & $5 \%(I)$ & $15 \%(3)$ & $20 \%(4)$ \\
\hline $\begin{array}{l}\text { Absent } \\
\text { (no visible imperfections caused by telangiectasia) }\end{array}$ & $0 \%(0)$ & $0 \%(0)$ & $0 \%(0)$ & $0 \%(0)$ \\
\hline
\end{tabular}

A progressive decrease of TEWL was noted throughout the treatment with Serum BK46, reaching a statistically significant difference in comparison with T0 following 14, 28 and 56 days of application. Indeed, as reported in the graph of Figure 3, the mean TEWL $\left(\mathrm{g} /\left(\mathrm{h}^{*} \mathrm{~m}^{2}\right)\right)$ observed at T0 was 15 \pm 3.1 , whereas at $\mathrm{T} 14, \mathrm{~T} 28$, and T56 it was respectively 14.3 $\pm 2.9 ; 12.9 \pm 2.7$, and $12.2 \pm 2.3$ ( $p$-value $<0.01$ vs $\mathrm{T} 0$, one way ANOVA, Student's $t$ post-test).

The improvement in the hydration parameters was accompanied by a reduction of skin erythema, in particular, a statistically significant decrease of erythema index was observed after 14, 28, and 56 days of Serum BK46 application, since baseline erythema index was $491 \pm 82.5$ at T0 but it dropped to $459.2 \pm 79.5,438.6 \pm 80.3$, and $425.4 \pm 78.7$ ( $p$-value $<0.05$ ) following 14,28 , and 56 days of application, respectively, as can be observed in the graph of Figure 4.
Furthermore, a slight, but statistically significant reduction of the capillaries' diameter was recorded following treatment; indeed, the median vessel diameter, measured at the level of arboreal telangiectasia, was 0.130 (Q1 0.081; $\mathrm{Q} 30.212) \mathrm{mm}$ at baseline (T0) and decreased to $0.119(\mathrm{Q} 1$ 0.068; Q3 0.188) $\mathrm{mm}$ and 0.112 (Q1 0.068; Q3 0.173) mm following, respectively, 28 and 56 days of product application: data are reported in the graph of Figure 5.

The reduction of erythema and telangiectasias was highlighted also by visual inspection at the end of treatment. Indeed, as reported in Figure 6, showing images taken at baseline and by the end of treatment, significant mitigation of both signs was highlighted by the reduction of superficial hemoglobin distribution following Serum BK46 application.

Clinical evaluation of skin redness and visibility of telangiectasia imperfections confirmed the effect of Serum 


\section{Skin moisturization}

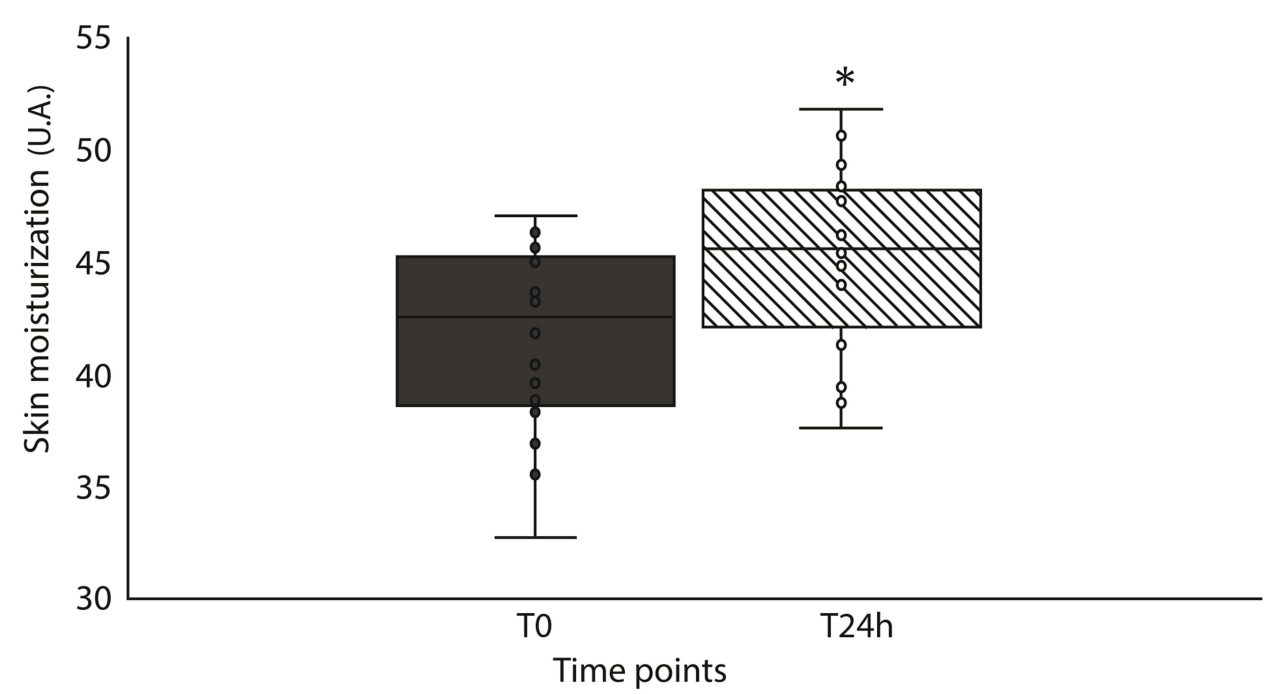

Figure I Short-term skin moisturization evaluated by a corneometer, reported as median \pm first and third quartiles at T0 and following $24 \mathrm{~h}$ of product application ( $\mathrm{T} 24 \mathrm{~h}$ ). $* p<0.01$ vs T0 Wilcoxon signed-rank test.

\section{Skin moisturization}

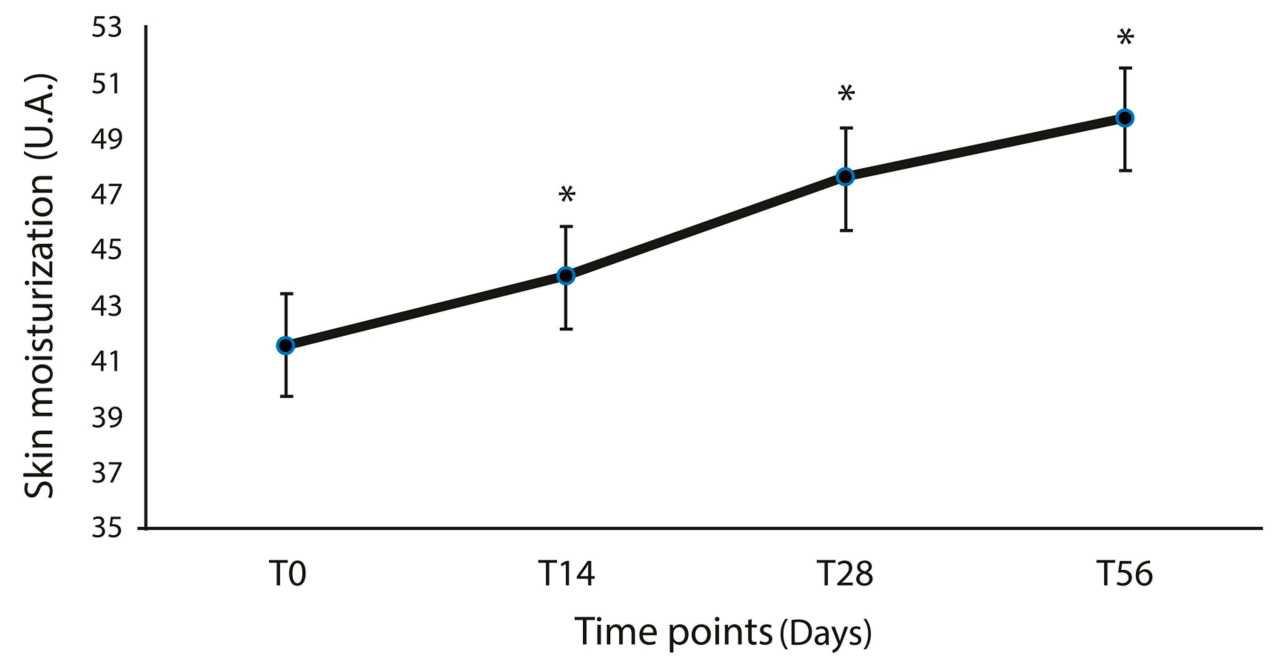

Figure 2 Long-term skin moisturization evaluated by a corneometer, reported as mean \pm standard error measured throughout the study, from T0 to T56. One-Way ANOVA test and Student's $t$ Bonferroni corrected post hoc test $\left({ }^{*} p<0.01\right.$ versus T0).

BK46 in reducing the main signs of rosacea, estimated according to the 5-point score shown in Table 1. In Table 2 is shown the comparison of clinical evaluation of rosacea symptoms (skin redness and telangiectasia imperfection visibility) score at baseline (before treatment $=\mathrm{T} 0$ ) and after 14, 28 , and 56 days of Serum BK46 application. Before treatment, the majority of patients $(85 \%)$ were graded as "severe" or "very severe" for skin redness, whereas this percentage declined to $60 \%$ and $50 \%$ following 28 and 56 days of Serum BK46 application, respectively. Statistical evaluation performed by the non-parametric Friedman test, followed by Wilcoxon signed-rank post-test to compare the variations among the different time points, underlined a statistically significant difference in comparison with baseline (T0) following 28 and 56 days of treatment $(p<0.05$ T28 vs T0; $p<0.01$ T56 vs T0). 


\section{TEWL}

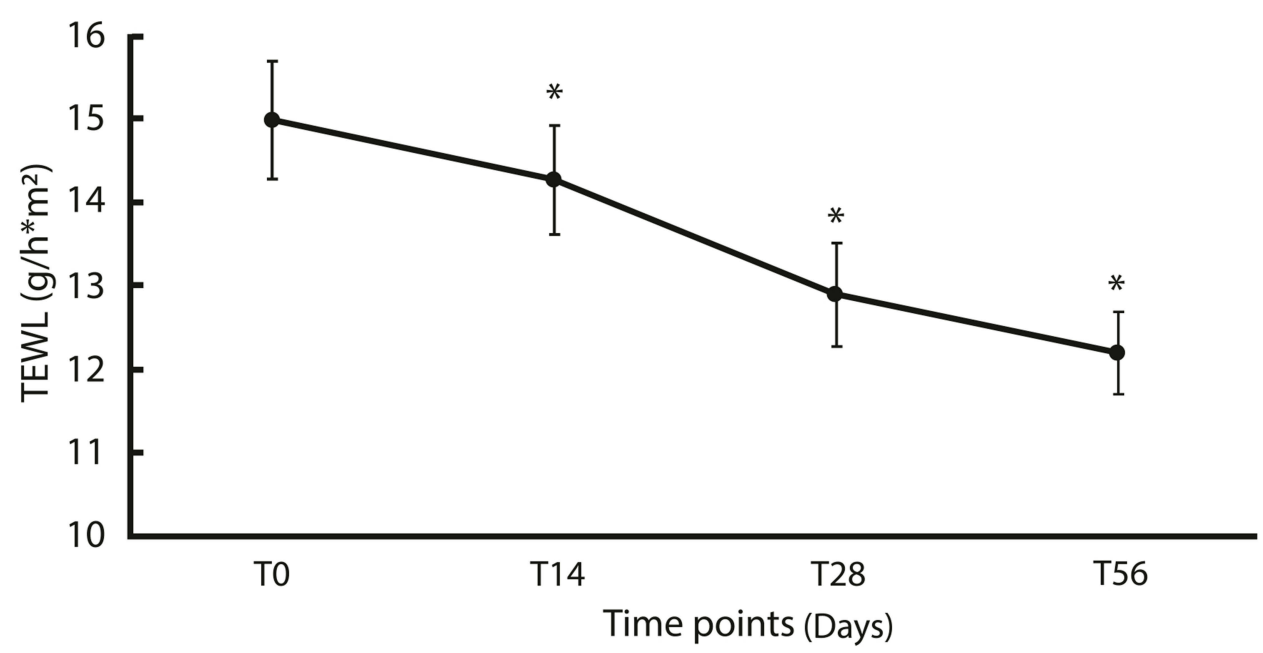

Figure 3 Long-term trans epidermal water loss (TEWL) measured by Tewameter. A regular decrease in the rate of skin dehydration was recorded during the study. Oneway ANOVA test and Student's $t$ Bonferroni corrected post hoc test $(* p<0.01$ vs T0).

\section{Skin erythema}

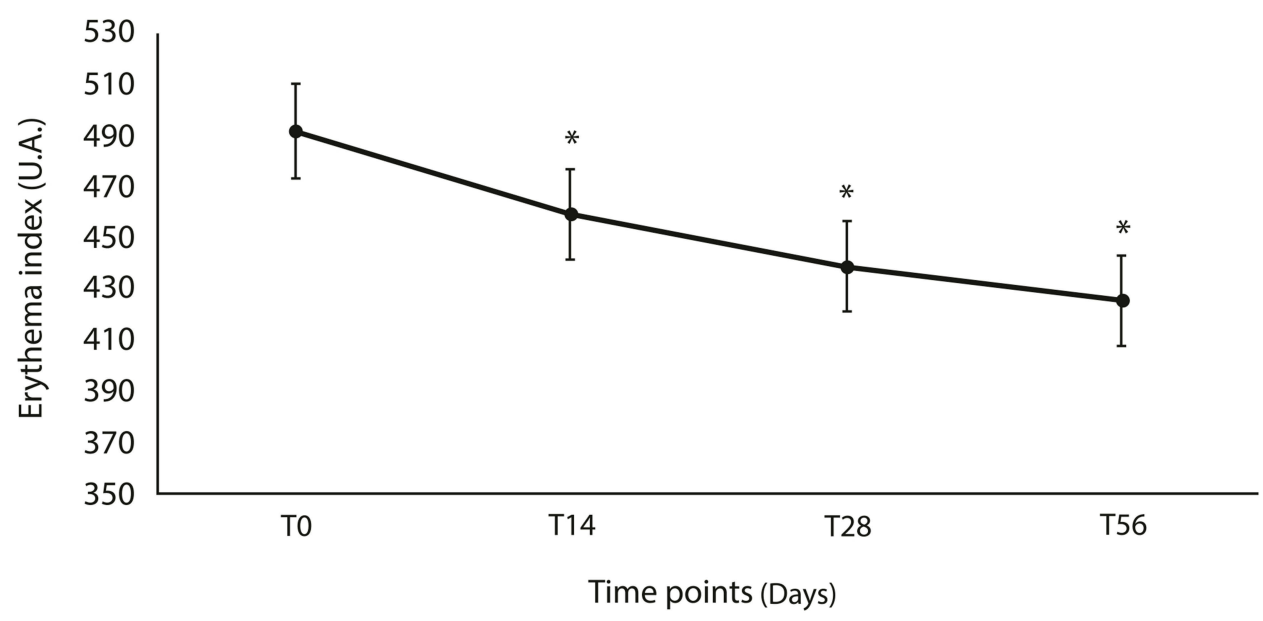

Figure 4 Skin erythema index as evaluated by Mexameter-MI8 throughout the study, the mean and standard error are reported. A steady decrease in skin associated erythema was observed. One-way ANOVA test and Student's $t$ Bonferroni corrected post hoc test $\left({ }^{*} p<0.0\right.$ I vs T0).

Similarly, a general reduction of telangiectasia imperfection visibility was observed by the end of treatment. In fact, the percentage of patients having severe or very severe imperfection visibility was $75 \%$ at baseline, but significantly declined to $60 \%$ and $55 \%$ after 28 and 56 days of product application. The difference between T56 and baseline was statistically significant, $p$-value $<0.01$.

\section{Discussion}

This study aims to evaluate the clinical effect of a Serum BK46, a product ideated for topical application for the treatment of the main signs of rosacea. In particular, the outcomes of long-term product application have been analyzed in patients with mild to moderate rosacea. Serum BK46 is a proprietary composition, including potassium azeloyl diglycinate, squalane, dipotassium glycyrrhizate, Aloe barbadensis leaf juice, sodium hyaluronate, polyacrylate crosspolymer-6, and xanthan gum; the efficacy of these ingredients against some of the rosacea signs has already been demonstrated. ${ }^{11,12}$

Rosacea is a chronic skin disorder that severely affects patient quality of life, since erythema, telangiectasia 


\section{Vessels diameter}

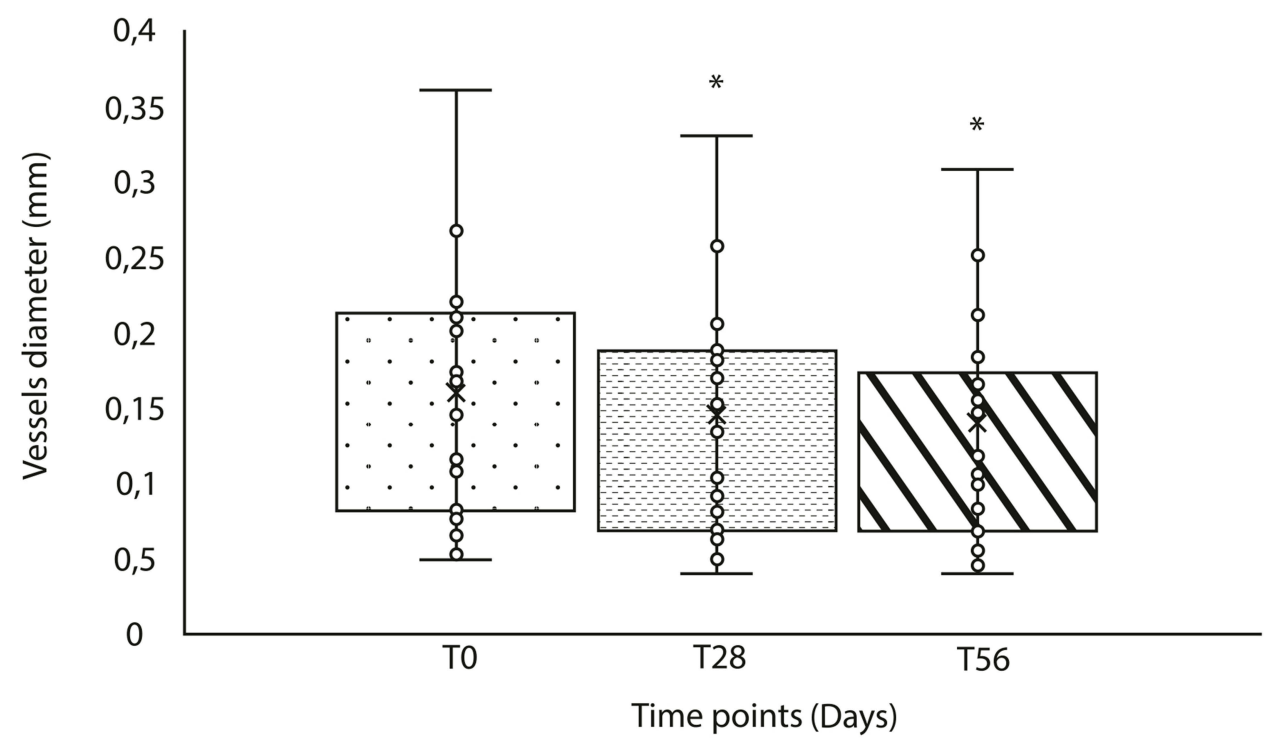

Figure 5 Vessel diameter as measured by Viodecap and reported as median with first and third quartile. ${ }^{*} p$-value $<0.01$ Friedman rank test, Wilcoxon-signed rank Bonferroni corrected post hoc test.

High hemoglobin concentration
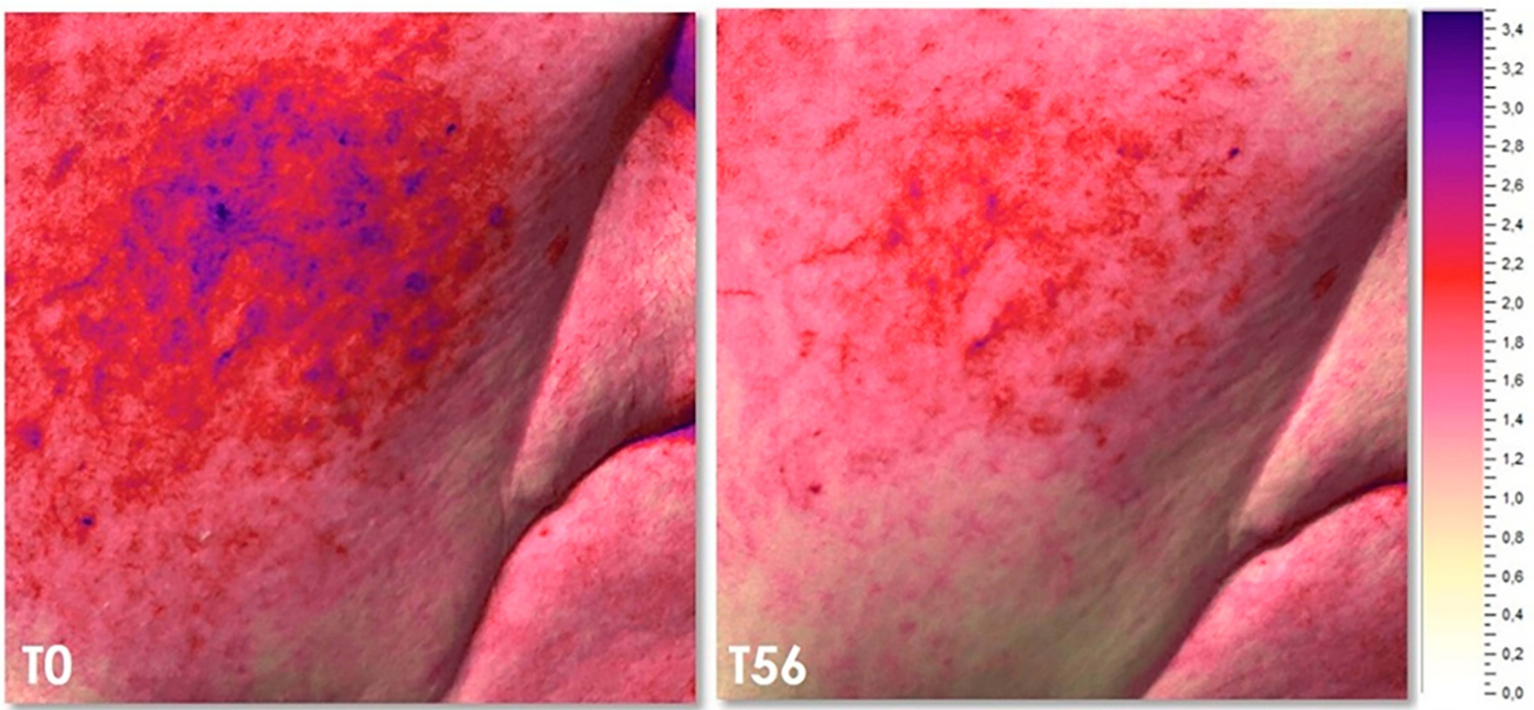

Low hemoglobin concentration

Figure 6 A sample image showing the reduction of superficial hemoglobin distribution in the cheek area at baseline and by the end of treatment, images detected and analyzed with Miravex Antera 3D.

imperfections, and other major symptoms mainly affect the facial skin, thus undermining patients' physical appearance. ${ }^{9}$ Indeed, patients usually suffer psychological discomfort and emotional stress due to the high visibility of pathology signs and this negative self-perception may lead to anxiety disorders.
Although a proper and well-defined cure for rosacea is far from being found, novel treatments that may control and relieve some symptoms may significantly improve patients' quality of life. Additionally, given the lack of an exhaustive treatment, a successful approach may be the avoidance of the triggering factors. 
The etiology of rosacea is still largely unknown; however, one of the triggering factors seems to be the damage to the skin barrier function. Indeed, one of the main functions of the skin is to provide an effective barrier to curtail the penetration of microbes and allergens into the body. Some studies have proven that alterations of skin barrier may promote skin disorders and defective expression of proteins involved in the correct setup of the skin barrier has been reported during rosacea progression. ${ }^{5,13}$ An altered barrier function is mainly visible by increased water loss, also promoted by the altered epidermal vascularization. ${ }^{5}$

Several clinical parameters have been used to assess the effect of Serum BK46 on symptom severity, including both instrumental techniques and clinical assessment to evaluate skin hydration, water loss, skin redness, erythema, and telangiectasias.

Serum BK46, due to its proprietary composition based on hydrating and skin protective agents, maintains skin hydration and restores the skin barrier function: a protective film is created over the skin, thus enforcing the cutaneous barrier and reducing the impact of environmental factors that could otherwise exacerbate the pathological signs. The data reported in this clinical study demonstrated that Serum BK46 application results in a diminished TEWL already after 14 days of treatment and the differences were statistically significant after 14 , 28 and 56 days if compared with baseline (T0). TEWL is the amount of water that evaporates through the skin and is used to describe skin barrier function as a shift from normal values to high levels indicates an impaired barrier functionality. In healthy subjects, TEWL of skin face reference value may be considered as $13 \mathrm{~g} /\left(\mathrm{h}^{*} \mathrm{~m}^{2}\right) .{ }^{14}$ The range of TEWL observed in rosacea patients enrolled in this study, as expected, was higher than the expected normal range, but long-term treatment with Serum BK46 could decrease TEWL to values in the normal range.

As a consequence of skin barrier restoration and due to the moisturizing agents contained, Serum BK46 also significantly increases skin hydration, which steadily rose throughout the treatment, reaching a marked increase following 14, 28, and 56 days of product application. These clinical findings have been confirmed also by patients' opinions, since in a self-administered questionnaire patients reported a marked reduction of dryness feeling following Serum BK46 treatment. This result is remarkable as the Serum BK46 application not only significantly relieves some of the main symptoms of rosacea, but it also improves the patient's self-perception about the impact of pathology-associated imperfections.

The restoration of skin hydration and the concomitant reduction of water loss would be of interest also to increase skin wound healing capability. ${ }^{15}$

The enhancement in skin hydration and barrier function is also accompanied by a normalization of the visibility of skin imperfections caused by telangiectasias. In particular, the instrumental measurements highlighted a progressive decrease in the visibility of superficial vessels and skin redness during treatment, reaching a statistically significant difference in comparison with baseline after 14, 28, and 56 days of Serum BK46 application. Skin hydration is one of the most important parameters to be evaluated in rosacea, for instance, several new treatments have been recently developed in order to improve the same parameters analyzed in this study. ${ }^{16,17}$

Furthermore, treatment with Serum BK46 significantly attenuated the visibility of telangiectasia imperfections, one of the most negative factors for facial perception associated with rosacea. In particular, both instrumental and clinical evaluation confirmed the decreased visibility of such dermal imperfection.

It is noteworthy that this reduction was evident during all the treatment periods, lasting 56 days; thus, strongly indicating that long-term product application could be an effective therapeutic strategy.

In addition, the product showed an optimal tolerability, as it did not induce any adverse effects and it could be applied safely for 8 weeks. Moreover, the majority of patients asked to state their opinions about the Serum BK46 effect judged the product effective in reducing skin redness and telangiectasia imperfection visibility (over 70\%) and the feeling of skin dryness and burning (over 75\%). The good rate of patient self-evaluation following Serum BK46 application is remarkable since, as stated above, a detrimental physical appearance perception is one of the primary factors worsening the quality of life for rosacea patients.

The preliminary results coming from this pilot study underline the clinical effect even in the long-term treatment of Serum BK46 in relieving some of the main rosacea symptoms. However, the power of this study is limited by the small number of subjects and by the absence of a control/placebo group; therefore, future studies should confirm these results in a larger group of patients including a placebo group or a split-face evaluation to provide even stronger evidence of Serum BK46 effectiveness. 


\section{Conclusion}

In conclusion, the results provided in this clinical study demonstrate the Serum BK46 effect in mitigating rosacea symptoms. Furthermore, its optimal tolerability even following long-term application makes Serum BK46 an ideal candidate for the long-term management of mild to moderate forms of rosacea.

\section{Ethics and Consent Statement}

This manuscript has not been published or submitted elsewhere for publication and it is not under consideration by another journal. The authors have read and understood the journal's policies and believe that neither the manuscript nor the study violates any of these.

\section{Disclosure}

The authors report no conflicts of interest in this work.

\section{References}

1. Wilkin J, Dahl M, Detmar M, et al. Standard grading system for rosacea: report of the National Rosacea Society Expert Committee on the classification and staging of rosacea. J. Am. Acad. Dermatol. 2004;50:907-912. doi:10.1016/j.jaad.2004.01.048

2. Rainer BM, Kang S, Chien AL. Rosacea: epidemiology, pathogenesis, and treatment. Dermatoendocrinol. 2017;9:e1361574. doi:10.1080/ 19381980.2017.1361574

3. Huynh TT. Burden of disease: the physchosocial impact of rosacea on a patient's quality of life. Am Health Drug Benefits. 2013;6(6):348-54.

4. Buddenkotte J, Steinhoff M. Recent advances in understanding and managing rosacea. F1000Research. 2018;7:1885. doi:10.12688/f100 Oresearch.16537.1

5. Addor FAS. Skin barrier in rosacea. An. Bras. Dermatol. 2016;91:59-63. doi:10.1590/abd1806-4841.20163541

6. Feaster B, Cline A, Feldman SR, Taylor S. Clinical effectiveness of novel rosacea therapies. Curr. Opin. Pharmacol. 2019;46:14-18. doi:10.1016/j.coph.2018.12.001
7. Korting HC, Schöllmann C. Current topical and systemic approaches to treatment of rosacea. J. Eur. Acad. Dermatol Venereol. 2009;23:876-882. doi:10.1111/j.1468-3083.2009.03167.x

8. Layton AM. Pharmacologic treatments for rosacea. Clin. Dermatol. 2017;35:207-212. doi:10.1016/j.clindermatol.2016.10.016

9. Baldwin H, Santoro F, Lachmann N, Teissedre S. A novel moisturizer with high sun protection factor improves cutaneous barrier function and the visible appearance of rosacea-prone skin. J. Cosmet. Dermatol. 2019;18(6):1686-1692. doi:10.1111/jocd.12889

10. Augustin M, Goepel L, Jacobi A, et al. Efficacy and tolerability of liposomal polyvinylpyrrolidone-iodine hydrogel for the localized treatment of chronic infective, inflammatory, dermatoses: an uncontrolled pilot study. Clin. Cosmet. Investig. Dermatol. 2017;10: 373-384. doi:10.2147/CCID.S141887

11. Schlesinger TE, Powell CR. Efficacy and tolerability of low molecular weight hyaluronic acid sodium salt $0.2 \%$ cream in rosacea. J. Drugs Dermatol. 2013;12:664-667.

12. Berardesca E, Iorizzo M, Abril E, et al. Clinical and instrumental assessment of the effects of a new product based on hydroxypropyl chitosan and potassium azeloyl diglycinate in the management of rosacea. J. Cosmet. Dermatol. 2012;11:37-41. doi:10.1111/j.14732165.2011.00598.x

13. Deng Z, Chen M, Xie H, et al. Claudin reduction may relate to an impaired skin barrier in rosacea. J. Dermatol. 2019;46:314-321. doi:10.1111/1346-8138.14792

14. Akdeniz M, Gabriel S, Lichterfeld-Kottner A, Blume-Peytavi U, Kottner J. Transepidermal water loss in healthy adults: a systematic review and meta-analysis update. Br J Dermatol. 2018;179:10 49-1055. doi:10.1111/bjd.17025

15. Zeng R, Lin C, Lin Z, et al. Approaches to cutaneous wound healing: basics and future directions. Cell Tissue Res. 2018;374:217-232. doi:10.1007/s00441-018-2830-1

16. Torregrosa A, Ochoa-Andrade AT, Parente ME, Vidarte A, Guarinoni G, Savio E. Development of an emulgel for the treatment of rosacea using quality by design approach. Drug Dev Ind Pharm. 2020;46(2):296-308. doi:10.1080/03639045.2020.1717515

17. Santoro F, Lachmann N. An open-label, intra-individual study to evaluate a regimen of three cosmetic products combined with medical treatment of Rosacea: cutaneous tolerability and effect on hydration. Dermatol Ther. 2019;9(4):775-784. doi:10.1007/s13555019-00331-4
Clinical, Cosmetic and Investigational Dermatology is an international, peer-reviewed, open access, online journal that focuses on the latest clinical and experimental research in all aspects of skin disease and cosmetic interventions. This journal is indexed on CAS.
The manuscript management system is completely online and includes a very quick and fair peer-review system, which is all easy to use. Visit http://www.dovepress.com/testimonials.php to read real quotes from published authors. 